\title{
A Cost-Effective Private-Key Cryptosystem for Color Image Encryption
}

\author{
Rastislav Lukac and Konstantinos N. Plataniotis \\ The Edward S. Rogers Sr. Dept. of Electrical and Computer Engineering, \\ University of Toronto, 10 King's College Road, Toronto, M5S 3G4, Canada \\ \{lukacr, kostas\}@dsp.utoronto.ca \\ http://www.dsp.utoronto.ca/ lukacr
}

\begin{abstract}
This paper presents a cost-effective private-key cryptosystem for color images. The scheme allows for secret sharing of the color image by generating two color shares with dimensions identical to those of the original. Encryption is performed via simple binary operations realized at the image bit-levels altering both the spectral correlation among the RGB color components and the spatial correlation between the neighboring color vectors. The decryption procedure uses both noise-like color shares as the input and recovers the original image with perfect reconstruction.
\end{abstract}

\section{Introduction}

Image secret sharing techniques [1],[2] represent a popular encryption tool used to secure transmission of personal digital photographs [3],[4] and digital documents [5] via public communication networks. The so-called $\{k, n\}$-threshold scheme [1],[4]-[9] encrypts the input image by splitting the original content into $n$ noise-like shares. The secret information is recovered only if $k$ or more shares are available for decryption [6]-[9].

Among the various $\{k, n\}$-threshold schemes, a simple $\{2,2\}$ solution, seen as a private-key cryptosystem [8],[9], takes a great popularity due to its simplicity and adequate information security. The encryption process splits the secret image into two noise-like shares which are delivered to the end-user independently. To recover the actual information the end-user should be in possession of both shares.

Although numerous secret sharing solutions have been proposed for encryption of binary and gray-scale images [1],[6]-[11], secret sharing of color images has become increasingly important in recent times. The growing interest in the development the color image encryption techniques [3]-[5],[12]-[17] can be attributed primarily to the proliferation of color imaging systems and imagingenabled consumer electronic devices such as digital cameras and mobile phones [18]. End-users and system developers have to protect personal digital photographs and scanned digital documents in emerging applications such as digital photo archiving and image transmission through wireless (mobile) networks. The 
surge of emerging applications and the proliferation of color imaging systems and imaging-enabled consumer electronic devices suggests that the demand for color image encryption solutions will continue.

\section{Color Imaging Basics}

Let us consider a $K_{1} \times K_{2}$ Red-Green-Blue (RGB) image $\mathbf{x}: Z^{2} \rightarrow Z^{3}$ representing a two-dimensional matrix of three-component vectorial inputs. In a given $K_{1} \times K_{2}$ RGB color image $\mathbf{x}$, pixel $\mathbf{x}_{(p, q)}=\left[x_{(p, q) 1}, x_{(p, q) 2}, x_{(p, q) 3}\right]$ denotes the color vector occupying the spatial location $(p, q)$, with $p=1,2, \ldots, K_{1}$ and $q=1,2, \ldots, K_{2}$ denoting the image row and column, respectively. The component $x_{(p, q) i}$, for $i=1,2,3$, of the RGB vector $\mathbf{x}_{(p, q)}$ denotes the vector's spectral component, namely: $x_{(p, q) 1}$ denotes the $\mathrm{R}$ component, $x_{(p, q) 2}$ denotes the $\mathrm{G}$ component, and $x_{(p, q) 3}$ indicates the B component.

Following the tristimulus theory of color representation, each color pixel $\mathbf{x}_{(p, q)}$ is a three-dimensional vector, uniquely defined by its length (magnitude) $M_{\mathbf{x}}$ : $Z^{2} \rightarrow R^{+}$and orientation (direction) $D_{\mathbf{x}}: Z^{2} \rightarrow S^{2}$ in the vector space, [18]. The magnitude

$$
M_{\mathbf{x}_{(p, q)}}=\left\|\mathbf{x}_{(p, q)}\right\|=\sqrt{x_{(p, q) 1}^{2}+x_{(p, q) 2}^{2}+x_{(p, q) 3}^{2}}
$$

of the color vector relates to the luminance, whereas the vectors' directionality

$$
D_{\mathbf{x}_{(p, q)}}=\frac{\mathbf{x}_{(p, q)}}{\left\|\mathbf{x}_{(p, q)}\right\|}=\frac{\mathbf{x}_{(p, q)}}{M_{\mathbf{x}_{(p, q)}}}
$$

with $S^{2}$ denoting a unit ball in $R^{3}$ and $\left\|D_{\mathbf{x}_{(p, q)}}\right\|=1$, relates to the chromaticity characteristics of the pixel.

Since both measures are essential for human perception [19], any color image encryption solution should alter both the magnitude and the orientation characteristics of the original color vectors. Using secret sharing principles for color image encryption [3]-[5],[13], the cryptographic solution generates color shares which contain noise-like, seemingly unrelated information.

\section{Overview of Color Image Secret Sharing Solutions}

Popular visual secret sharing (VSS) schemes, such as those proposed in [1],[6]-[9], allow for visual recovery of the encrypted images [1]. By utilizing the transparent/frosted representation of the shares (usually printed on transparencies) and color mixing principles, the color VSS schemes such as those listed in [12],[14],[15] use the properties of the human visual system (HVS) to force the recognition of a secret message from the required set of shares without additional computations or any knowledge of cryptography [1]. On the other hand, VSS schemes can also be implemented via simple logical operations and used in a computercentric environment. Although these features make the VSS decryption system 
(a)

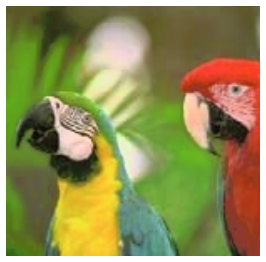

(b)

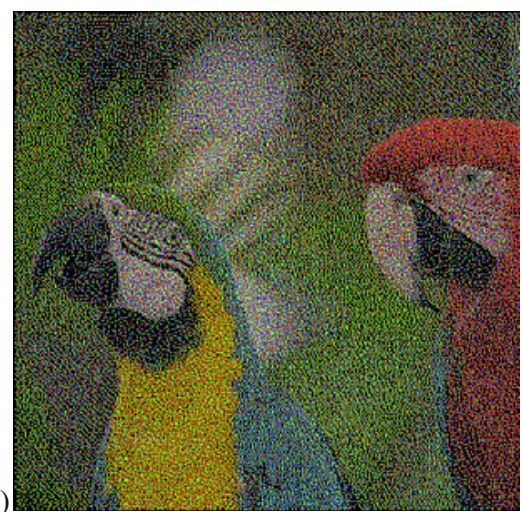

(c)

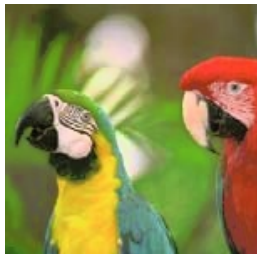

Fig. 1. Obtained results: (a) color test image Parrots, (b) the decrypted output obtained using the VSS scheme, (c) the decrypted output obtained using the bit-level processing based secret sharing scheme

cost-effective, such an approach is not well suited for natural color images. This is due to the fact that the procedure increases the spatial dimension of both shares and output image and introduces a number of visual impairments to the output image (Fig.1b).

The secret sharing schemes of [3]-[5] operate on the bit planes of the digital input and generate the shares with a bit representation identical to the one of the input image. Although the input image and the produced shares have different spatial dimensions due to encryption of each pixel into the blocks of share pixels, the decryption procedure produces an output image (Fig.1c) which is identical to the input (Fig.1a). It was explained in [13] that such a cryptographic solution satisfies the so-called perfect reconstruction property. Since both bit-level processing based $\{k, n\}$-solutions [3]-[5], and $\{2,2\}$ private key cryptosystem of [13] increase the dimensions of the shares compared to those of the original (secret) image, the produced shares with the enlarged spatial resolution may become difficult to transmit via wireless mobile networks.

\section{Private-Key Cryptosystem for Color Image Encryption}

To avoid this drawback, our new private-key cryptosystem for color image encryption reduces the block-based share operations to the pixel-based operations [16]. Thus, the spatial resolution of the shares remain unchanged during processing. Moreover, since the scheme performs cryptographic processing on bit planes, it: i) preserves the bit-representation of the shares, and ii) produces an output image identical to the input image. This suggests that the scheme recovers the input image with perfect reconstruction.

Assuming the conventional RGB color image, each color component $x_{(p, q) k}$ of the color vector $\mathbf{x}_{(p, q)}$ is coded with $B=8$ bits allowing $x_{(p, q) k}$ to take an 


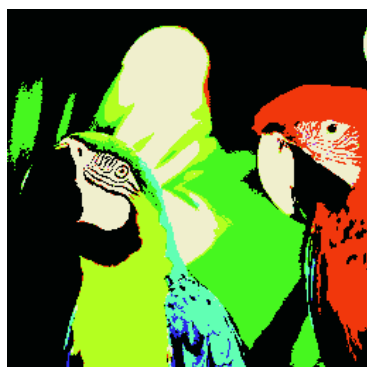

(a)

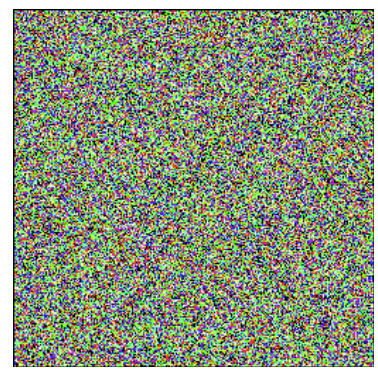

(b)

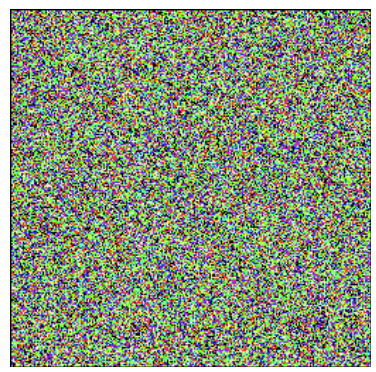

(c)

Fig. 2. Encryption of the MSB $(b=1)$ plane: (a) the original input image $\mathbf{x}^{b},(\mathrm{~b})$ the share $\mathbf{s}^{\prime b},(\mathrm{c})$ the share $\mathbf{s}^{\prime \prime b}$

integer value between 0 and $2^{B}-1$. Using a bit-level representation [13], the color vector $\mathbf{x}_{(p, q)}$ can be equivalently expressed in a binary form as follows:

$$
\mathbf{x}_{(p, q)}=\sum_{b=1}^{B} \mathbf{x}_{(p, q)}^{b} 2^{B-b}
$$

where $\mathbf{x}_{(p, q)}^{b}=\left[x_{(p, q) 1}^{b}, x_{(p, q) 2}^{b}, x_{(p, q) 3}^{b}\right] \in\{0,1\}^{3}$ denotes the binary vector at the $b$-bit level, with $b=1$ denoting the most significant bit (MSB).

Since the proposed private-key cryptosystem is a simple $\{2,2\}$-secret sharing scheme, binary vectors $\mathbf{x}_{(p, q)}^{b}$, for $b=1,2, \ldots, B$, are encrypted into two binary share vectors $\mathbf{s}_{(p, q)}^{\prime b}=\left[s_{(p, q) 1}^{\prime b}, s_{(p, q) 2}^{\prime b}, s_{(p, q) 3}^{\prime b}\right]$ and $\mathbf{s}_{(p, q)}^{\prime \prime b}=\left[s_{(p, q) 1}^{\prime \prime b}, s_{(p, q) 2}^{\prime \prime b}, s_{(p, q) 3}^{\prime \prime b}\right]$, whose components $s_{(p, q) k}^{\prime b}$ and $s_{(p, q) k}^{\prime \prime b}$ are generated as follows [16]:

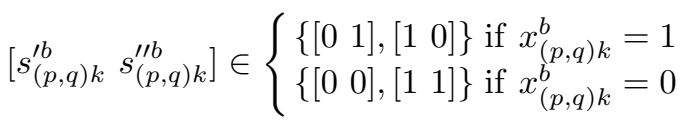

where the binary set $\left[s_{(p, q) k}^{\prime b} s_{(p, q) k}^{\prime \prime b}\right]$ is obtained from the basis elements 0 and 1 .

To ensure the random nature of the encryption (4), a random process should be guided by a random number generator. In this paper, we use the conventional rand function that is built in to common $\mathrm{C}++$ programming tools to determine $\left[s_{(p, q) k}^{\prime b} s_{(p, q) k}^{\prime \prime b}\right]$ from the sets $\left\{\left[\begin{array}{ll}0 & 1\end{array}\right],\left[\begin{array}{ll}1 & 0\end{array}\right]\right\}$ and $\left\{\left[\begin{array}{ll}0 & 0\end{array}\right],\left[\begin{array}{ll}1 & 1\end{array}\right]\right\}$ via $(4)$. By repeating the process at each binary level $b=1,2, \ldots, B$ and each vector component $k=1,2,3$, the procedure generates two color share vectors $\mathbf{s}_{(p, q)}^{\prime}=\left[s_{(p, q) 1}^{\prime}, s_{(p, q) 2}^{\prime}, s_{(p, q) 3}^{\prime}\right]$ and $\mathbf{s}_{(p, q)}^{\prime \prime}=\left[s_{(p, q) 1}^{\prime \prime}, s_{(p, q) 2}^{\prime \prime}, s_{(p, q) 3}^{\prime \prime}\right]$ defined as follows:

$$
\mathbf{s}_{(p, q)}=\sum_{b=1}^{B} \mathbf{s}_{(p, q)}^{\prime b} 2^{B-b}, \mathbf{s}_{(p, q)}^{\prime \prime}=\sum_{b=1}^{B} \mathbf{s}_{(p, q)}^{\prime{ }^{b}} 2^{B-b}
$$

where $\mathbf{s}_{(p, q)}^{\prime b}$ and $\mathbf{s}_{(p, q)}^{\prime \prime b}$ denote the binary share vectors obtained in (4). As shown in Fig. 2 the formation of the binary share vector arrays increases the degree 


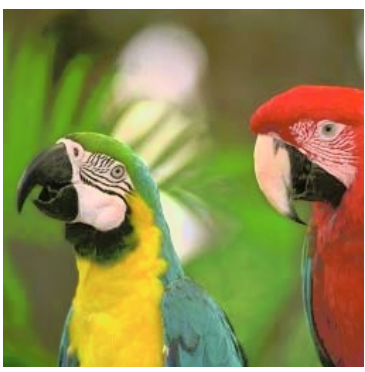

(a)

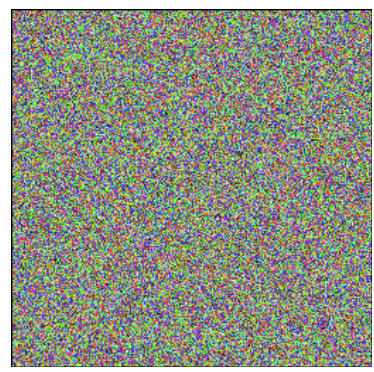

(b)

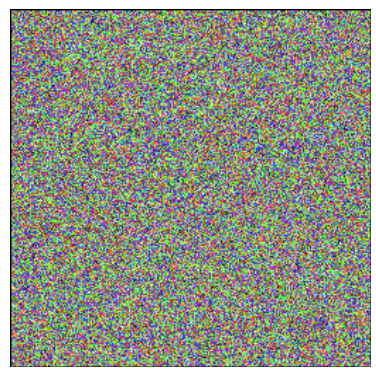

(c)

Fig. 3. Proposed color image encryption: (a) the input (original) color image $\mathbf{x}$, (b) the color share $\mathbf{s}^{\prime},(\mathrm{c})$ the color share $\mathbf{s}^{\prime \prime}$

of protection from two possible options in (4) to eight options observed for the binary share vectors $\mathbf{s}_{(p, q)}^{\prime b}$ and $\mathbf{s}_{(p, q)}^{\prime \prime b}$ at each bit level $b$.

By performing the processing operations (3)-(5) in each spatial location $(p, q)$, for $p=1,2, \ldots, K_{1}$ and $q=1,2, \ldots, K_{2}$, the procedure produces two $K_{1} \times K_{2}$ color shares $\mathbf{s}^{\prime}: Z^{2} \rightarrow Z^{3}$ and $\mathbf{s}^{\prime \prime}: Z^{2} \rightarrow Z^{3}$, with the share vectors $\mathbf{s}^{\prime}(p, q)$ and $\mathbf{s}^{\prime \prime}{ }_{(p, q)}$ denoting the color pixels located at $(p, q)$ in $\mathbf{s}^{\prime}$ and $\mathbf{s}^{\prime \prime}$, respectively. This increases protection of both the individual color channels $\left(2^{B}\right.$ levels $)$ and the color shares ( $2^{3 B}$ possible color vectors) depicted in Figs.3b, $c$ since the random binary vectors $\mathbf{s}_{(p, q)}^{\prime b}$ and $\mathbf{s}_{(p, q)}^{\prime \prime b}$ are weighted by $2^{B-b}$ needed in the determination of the word-level color image model.

It is shown in [16] that due to the randomness in the encryption process defined in (4) the color share vectors $\mathbf{s}_{(p, q)}^{\prime}$ and $\mathbf{s}^{\prime \prime}{ }_{(p, q)}$ differ both in magnitude $\left(M_{\mathbf{s}^{\prime}(p, q)} \neq M_{\mathbf{s}^{\prime \prime}(p, q)}\right)$ and in direction $\left(D_{\mathbf{s}^{\prime}(p, q)} \neq D_{\mathbf{s}^{\prime \prime}(p, q)}\right)$. In addition, they differ in both magnitude and direction from those $\left(M_{\mathbf{x}_{(p, q)}}, D_{\mathbf{x}_{(p, q)}}\right)$ observed for the original color inputs $\mathbf{x}_{(p, q)}$. As it can be seen in Fig. 3 the encryption process changes the spectral correlation characteristics of the input vectors and alters the spatial correlation characteristics of the input image. The utilization of the complete RGB color gamut $\left(256^{3}=16,777,216\right.$ colors $)$ in the proposed encryption procedure i) ensures adequate protection against attacks in the color share domain, and ii) prohibits unauthorized access to the original bit information.

To faithfully decrypt the secret color image from the color shares, the decryption function must satisfy the perfect reconstruction property. This can be obtained when the encryption and decryption operations are reciprocal [13]. Therefore, the original color/structural information is recovered by processing the share vector arrays at the binary level, and the decryption function follows the encryption mechanism in (4) resulting in the following definition [16]:

$$
x_{(p, q) k}^{b}=\left\{\begin{array}{l}
0 \text { if } s_{(p, q) k}^{\prime b}=s_{(p, q) k}^{\prime \prime b} \\
1 \text { if } s_{(p, q) k}^{\prime b} \neq s_{(p, q) k}^{\prime \prime b}
\end{array}\right.
$$

Based on the reciprocal concept between (4) and (6), the original binary component $x_{(p, q) k}^{b}$ is recover as $x_{(p, q) k}^{b}=1$ if the binary share components 


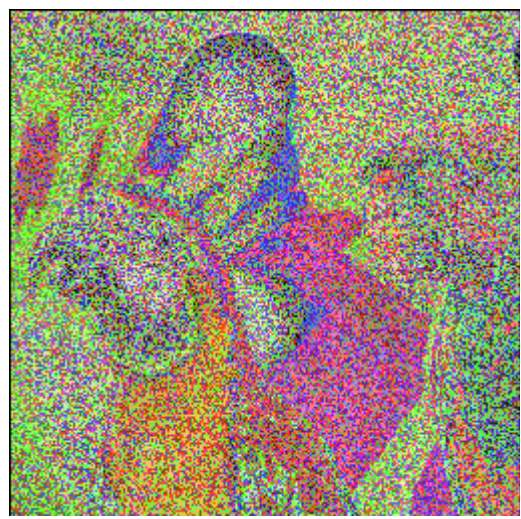

(a)

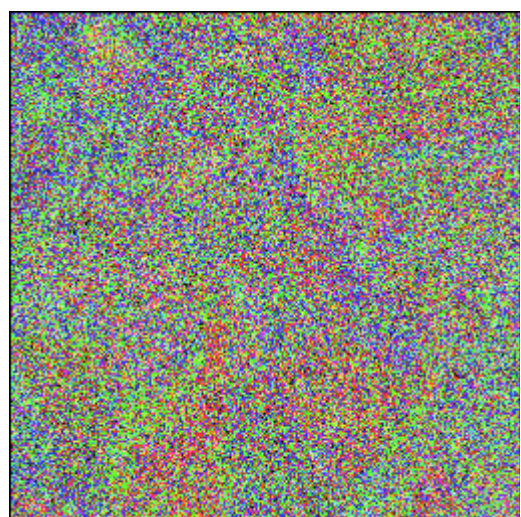

(b)

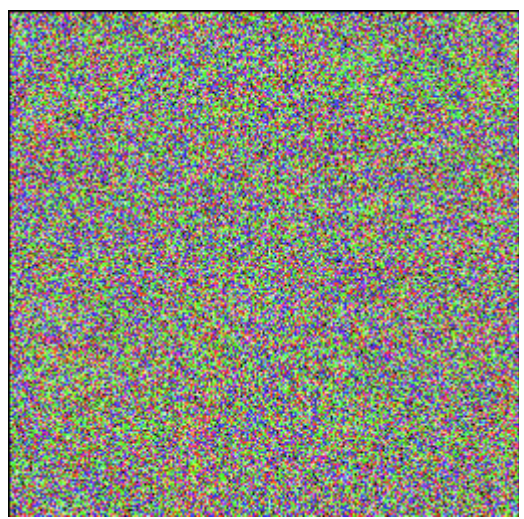

(c)

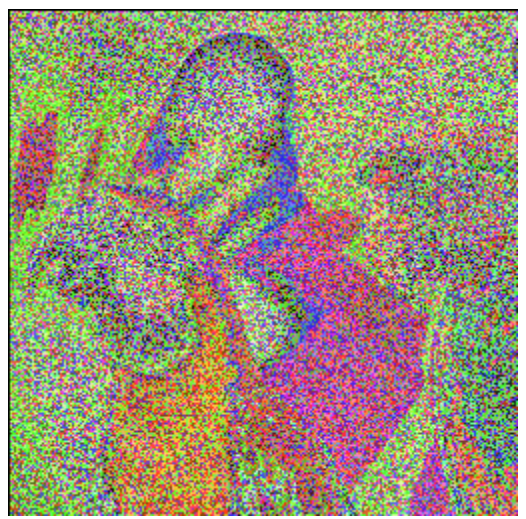

(d)

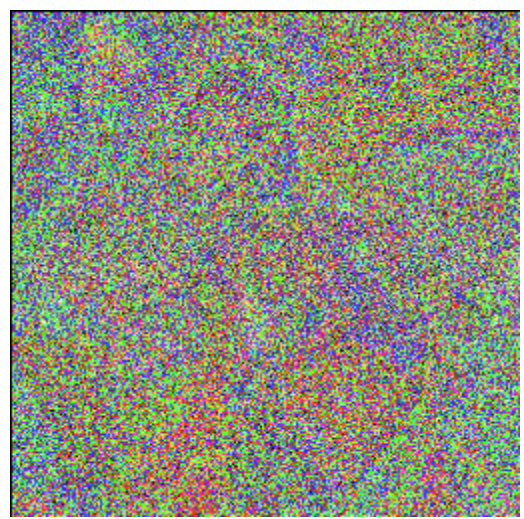

(e)

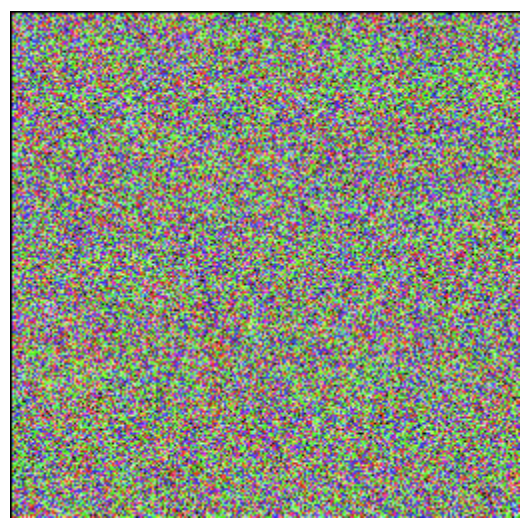

(f)

Fig. 4. Color shares $\mathbf{s}^{\prime}(\mathrm{a}-\mathrm{c})$ and $\mathbf{s}^{\prime \prime}$ (d-f) obtained using the color image Parrots when cryptographic processing is performed for the reduced set of binary levels: (a,d) $b=1$, (b,e) $b=1,2,(\mathrm{c}, \mathrm{f}) b=1,2,3$ 
$s_{(p, q) k}^{\prime b}$ and $s_{(p, q) k}^{\prime \prime b}$ are not equal or $x_{(p, q) k}^{b}=0$ if $s_{(p, q) k}^{\prime b}$ and $s_{(p, q) k}^{\prime \prime b}$ are identical. Stacking together the recovered bits $x_{(p, q) k}^{b}$ using the spectral relationship $\mathbf{x}_{(p, q)}^{b}=\left[x_{(p, q) 1}^{b}, x_{(p, q) 2}^{b}, x_{(p, q) 3}^{b}\right]$ the binary vector $\mathbf{x}_{(p, q)}^{b}$ is formed. Application of (3) results in the recovered original color vector $\mathbf{x}_{(p, q)}$, which suggests that the proposed method satisfies the perfect reconstruction property.

Figs.3b,c show the color shares obtained when the cryptographic operations are applied to all bit planes $(b=1,2, \ldots, B)$. The complexity of the solution can be reduced by performing the encryption/decryption operations for a reduced set of bit planes. Visual inspection of the color shares depicted in Fig. 4 reveals that the encryption of the MSB (Figs.4a,d) or the two most significant bits (Figs.4b,e) only, fine details are sufficiently encrypted, however, large flat regions can be visually revealed. The results depicted in Figs.4c,f indicate that a sufficient level of protection is achieved by cryptographically processing the first three most significant bits $(b=1,2,3)$. The remaining bits of the original image vectors can be simply copied into the shares unchanged. If this option is selected, image decryption has to be performed only for $b=1,2,3$.

\section{Conclusion}

A private-key cryptosystem for color image encryption was presented. The solution can be seen a $\{2,2\}$-secret sharing scheme which satisfies the perfect reconstruction property. By applying simple logical cryptographic operations at the bit-level, the encryption procedure: i) changes both the magnitude and the orientation of the color vectors generating color noise-like shares, and ii) it produces random-like color vectors which differ significantly in both magnitude and orientation from the original color inputs. Thus, the generated shares which can be transmitted over unsecured public channels with reasonable overhead. Since the method performs the pixel-based share operations instead of the usual block-based operations, the produced color hares have the same spatial resolution as the original image. The input color image is perfectly reconstructed from the share vector arrays using elementary bit-level logical functions. The perfect reconstruction allowed by the procedure makes it ideal for cost-effective dissemination of digital imaging material over untrusted communication channels.

\section{References}

1. Naor, M., Shamir, A.: Visual Cryptography. Proc. EUROCRYPT'94, LNCS 950 (1994) 1-12

2. Yang, C.N., Laih, C.S.: New colored visual secret sharing schemes. Designs Codes and Cryptography 20 (2000) 325-336

3. Lukac, R., Plataniotis, K.N., Smolka, B., Venetsanopoulos, A.N.: A new approach to color image secret sharing. Proc. XII European Signal Processing Conference (EUSIPCO'04) in Vienna, Austria, (2004) 1493-1496 
4. Lukac, R., Plataniotis, K.N.: A color image secret sharing scheme satisfying the perfect reconstruction property. Proc. 2004 IEEE International Workshop on Multimedia Signal Processing (MMSP'04) in Sienna, Italy, (2004) 351-354

5. Lukac, R., Plataniotis, K.N.: Document image secret sharing using bit-level processing. Proc. 2004 IEEE International Conference on Image Processing (ICIP'04) in Singapore, (2004) 2893-2896

6. Lin, C.C., Tsai, W.H., Visual cryptography for gray-level images by dithering techniques. Pattern Recognition Letters 24 (2003) 349-358

7. Chang, C.C, Chuang, J.C.: An image intellectual property protection scheme for gray-level images using visual secret sharing strategy. Pattern Recognition Letters, 23 (2002) 931-941

8. Lukac, R., Plataniotis, K.N.: Bit-level based secret sharing for image encryption. Pattern Recognition 38 (2005) 767-772

9. Ateniese, G., Blundo, C, de Santis, A., Stinson, D.G.: Visual cryptography for general access structures. Information and Computation 129 (1996) 86-106

10. Yang, C.N.: New visual secret sharing schemes using probabilistic method. Pattern Recognition Letters, 25 (2004) 481-494

11. Yang, C.N., Chen, T.S: Aspect ratio invariant visual secret sharing schemes with minimum pixel expansion. Pattern Recognition Letters, 26 (2005) 193-206.

12. Hou, J.C.: Visual cryptography for color images. Pattern Recognition 36 (2003) 1619-1629

13. Lukac, R., Plataniotis, K.N.: Colour image secret sharing. IEE Electronics Letters 40 (2004) 529-530

14. Koga, H., Iwamoto, M., Yakamoto, H.: An analytic construction of the visual secret sharing scheme for color images. IEICE Transactions on Fundamentals E84-A (2001) 262-272

15. Ishihara, T., Koga, H.: A visual secret sharing scheme for color images based on meanvalue-color mixing. IEICE Transactions on Fundamentals E86-A (2003) 194197

16. Lukac, R., Plataniotis, K.N.: A new encryption scheme for color images. Computing and Informatics, submitted (2004)

17. Sudharsanan, S.: Visual cryptography for JPEG color images. Proceedings of the SPIE, Internet Multimedia and Management Systems V, 5601 (2004) 171-178

18. Lukac, R., Smolka, B., Martin, K., Plataniotis, K.N., Venetsanopoulos, A.N.: Vector filtering for color imaging. IEEE Signal Processing Magazine, Special Issue on Color Image Processing 22 (2005) 74-86

19. Sharma, G., Trussell, H.J.: Digital color imaging. IEEE Transactions on Image Processing 6 (1997) 901-932 